\title{
Phase space measure concentration for an ideal gas
}

\author{
ANTHONY J. CREACO*, NIKOS KALOGEROPOULOS ${ }^{\dagger}$ \\ Department of Science \\ BMCC-The City University of New York \\ 199 Chambers St., New York, NY 10007, USA
}

\begin{abstract}
We point out that a special case of an ideal gas exhibits concentration of the volume of its phase space, which is a sphere, around its equator in the thermodynamic limit. The rate of approach to the thermodynamic limit is determined. Our argument relies on the spherical isoperimetric inequality of Lévy and Gromov.
\end{abstract}

PACS: $\quad$ 02.30. $\mathrm{Cj}, \quad 02.40 . \mathrm{Ky}, \quad 05.20 . \mathrm{Gg}$

Keywords: Ideal gas, Measure concentration, Isoperimetric inequalities.

\footnotetext{
E-mail: $\quad *$ acreaco@bmcc.cuny.edu

† nkalogeropoulos@bmcc.cuny.edu,nkaloger@yahoo.com
} 


\section{IntroduCtion}

The concentration of measure phenomenon [1] is an important concept in contemporary analysis and measure theory. It was very effectively used by V.D. Milman in his proof of Dvoretzky's theorem [2] on the existence of almost spherical sections of convex bodies and by many others ever since [1]. Roughly speaking, the concentration of measure states as the dimension of the underlying geometric structure, be it a subset of Euclidean or a Banach space, a manifold, a metric space etc, approaches infinity, most of the measure considered in such a structure can be found concentrated in a subset of it. Such a subset would be considered "small" by the casual observer. Dually, measure concentration can be seen as a property of functions of a large number of variables with small oscillations which turn out to be almost constant. This interpretation goes back to P. Lévy [3]. The geometric concept of "observable diameter" and related quantities introduced in [4] is also motivated by this observation.

Isoperimetric inequalities are some of the most studied geometric inequalities [5], especially during the last century and a half. Roughly speaking, isoperimetric inequalities provide bounds to the relation between the volume of a domain and the area of its boundary. A physical motivation is to prove the observation that the shape of a droplet, or bubble, in ordinary flat space is spherical and to explore what this optimal shape would be in other situations such as Riemannian spaces of constant or variable curvature, metric-measure spaces etc. Despite the best efforts of decades, such minimizers are known only in the case of the Euclidean space $\mathbb{R}^{n}[6]$, the sphere $S^{n}[7]$ and the hyperbolic space $H^{n}[8]$ equipped with their usual metrics of constant sectional curvature $0,+1$ and -1 respectively. The concentration of measure phenomenon is controlled through inequalities, whose geometric incarnation has a strong isoperimetric flavor. The present work use the fact that spherical caps are the isoperimetric domains/minmizers in $S^{n}[7]$.

In this letter we present an application of the measure concentration on $S^{n}$ and the related isoperimetric inequality of P. Lévy [3] which was generalized much later by M. Gromov [7], to a particular case of an ideal gas. To be more specific, we consider an ideal non-relativistic gas which is placed inside a one-dimensional thermally isolated box, i.e. inside a line segment. We assume that initially this gas contains $n-1$ (point) particles/molecules. We then add one extra particle/molecule which is stationary in the frame of reference of the box. The total energy of the system remains the same before 
and after we add this $n$-th particle. We continue this process, ad infinitum, and we try to understand the behavior of the volume of the phase space of the system in such a limit. The phase space has a compact part which is a sphere. We find that as $n$ increases, the volume of such a sphere concentrates around its equator The result that we find is that, in the thermodynamic limit $n \rightarrow \infty$, the micro-canonical partition function of the gas receives its dominant contribution from a tubular neighborhood of the equator of width proportional to $n^{-\frac{1}{2}}$. Thus, we provide a geometric description of the system and, through it, an expression for the rate at which the system approaches equilibrium. This letter should be considered as a counterpart of [11] where a similar type of arguments was used to determine the rate of approach to equilibrium of a parity-violating system.

In Section 2, we start the mathematical description of such a system by calculating the relative volume of a tubular neighborhood of the equator of a sphere and compare it to the volume of the sphere itself. We find that, to a first order approximation, that this ratio is zero, as is intuitively expected. In Section 3 we show how the situation changes dramatically when a more careful, second order, approximation to such a ratio is made. Here the concentration of measure becomes manifest. In Section 4 we discuss how Sections 2 and 3 can be applied to the specific case of the ideal gas that we are considering. Section 5 contains some further comments and some conclusions that can be drawn from such an analysis.

\section{Relative VOLUMES OF SPHERES; FIRST ORDER APPROXIMATION}

Consider a system of $n+1$ non-relativistic particles on a line. The phase space of such a system is $\mathbb{R}^{n+1} \times \mathbb{R}^{n+1}$. If the total energy $E$ of the system is conserved, and assume that, for any reason, the location of the individual particles does not affect the value of $E$, then the phase space reduces to $S^{n} \times \mathbb{R}^{n+1}$. We focus our attention on the compact part of the phase space, the $n$-sphere $S^{n}$, because, eventually, the contribution of the potential function, which depends on the generalized coordinates only, will turn out to be a constant in the micro-canonical partition function. Such a constant will not be relevant to our arguments, so we will eventually disregard it. We assume, for simplicity, that $S^{n}$ is equipped with its round metric $\rho$ induced from the Euclidean metric of $\mathbb{R}^{n+1}$. It is well-known that the geodesic segment joining $x_{1}, x_{2} \in S^{n}$ is an arc of a great circle of $S^{n}$. Let the corresponding convex angle subtended by such a geodesic arc at the center of the 
sphere be $\psi$. Then

$$
\rho\left(x_{1}, x_{2}\right)=\psi
$$

The Euclidean distance in the ambient space $\mathbb{R}^{n+1}$ is expressed in terms of $\psi$ as

$$
\left\|x_{1}-x_{2}\right\|=2 \sin \frac{\psi}{2}
$$

and the relation between $\rho\left(x_{1}, x_{2}\right)$ and $\left\|x_{1}-x_{2}\right\|$ is

$$
\frac{2}{\pi} \rho\left(x_{1}, x_{2}\right) \leq\left\|x_{1}-x_{2}\right\| \leq \rho\left(x_{1}, x_{2}\right)
$$

Let $d \mu$ denote the infinitesimal Riemannian measure on $S^{n}$ induced from the Lebesgue measure of $\mathbb{R}^{n+1}$. We have assumed that $S^{n}$ is trivially embedded in $\mathbb{R}^{n+1}$, which is parametrized by $x^{i}, i=1, \ldots, n+1, \quad x^{i} \in \mathbb{R}$ and $S^{n}$ is parametrized by the standard spherical coordinates $\theta_{i}, i=1, \ldots, n$ with $0 \leq \theta_{j}<\pi, j=1, \ldots, n-1, \quad 0 \leq \theta_{n}<2 \pi$ namely

$$
\begin{aligned}
x^{1} & =\sin \theta_{1} \sin \theta_{2} \cdots \sin \theta_{n-1} \sin \theta_{n} \\
x^{2} & =\sin \theta_{1} \sin \theta_{2} \cdots \sin \theta_{n-1} \cos \theta_{n} \\
& \vdots \\
x^{n} & =\sin \theta_{1} \cos \theta_{2} \\
x^{n+1} & =\cos \theta_{1}
\end{aligned}
$$

The Jacobian of (4) gives

$$
d \mu=d \theta_{n} \prod_{j=1}^{n-1} \sin ^{n-j} \theta_{j} d \theta_{j}
$$

Let $U_{\epsilon / 2}\left(S^{n-1}\right)$ denote an $\epsilon / 2$-neighborhood of the equator $S^{n-1} \subset S^{n}$ namely

$$
U_{\epsilon / 2}\left(S^{n-1}\right)=\left\{x \in S^{n}: \rho\left(x, S^{n-1}\right)<\frac{\epsilon}{2}\right\}
$$

and let $\operatorname{vol}\left(U_{\epsilon / 2}\left(S^{n-1}\right)\right)$ be its volume obtained from $d \mu$. Then

$$
\operatorname{vol}\left(U_{\epsilon / 2}\left(S^{n-1}\right)\right)=2 \pi \int_{\frac{\pi-\epsilon}{2}}^{\frac{\pi+\epsilon}{2}} \sin ^{n-1} \theta_{1} d \theta_{1} \prod_{j=2}^{n-1} \int_{0}^{\pi} \sin ^{n-j} \theta_{j} d \theta_{j}
$$

which gives

$$
\operatorname{vol}\left(U_{\epsilon / 2}\left(S^{n-1}\right)\right)=\operatorname{vol}\left(S^{n-1}\right) \int_{\frac{\pi-\epsilon}{2}}^{\frac{\pi+\epsilon}{2}} \sin ^{n-1} \theta_{1} d \theta_{1}
$$


Setting $\phi=\theta_{1}-\frac{\pi}{2}$, and using the fact that $\cos \phi$ is an even function on $\mathbb{R}$, we find

$$
\operatorname{vol}\left(U_{\epsilon / 2}\left(S^{n-1}\right)\right)=\operatorname{vol}\left(S^{n-1}\right) 2 \int_{0}^{\frac{\epsilon}{2}} \cos ^{n-1} \phi d \phi
$$

We are interested in determining the ratio

$$
\frac{\operatorname{vol}\left(U_{\epsilon / 2}\left(S^{n-1}\right)\right)}{\operatorname{vol}\left(S^{n}\right)}=\frac{\operatorname{vol}\left(S^{n-1}\right)}{\operatorname{vol}\left(S^{n}\right)} 2 \int_{0}^{\frac{\epsilon}{2}} \cos ^{n-1} \phi d \phi
$$

To complete the calculation we have to determine $2 \int_{0}^{\frac{\epsilon}{2}} \cos ^{n-1} \phi d \phi$. We can evaluate it recursively or we can prove by induction, a posteriori, that [12] for $m \in \mathbb{N}$,

$$
\int_{0}^{\frac{\epsilon}{2}} \cos ^{2 m+1} \phi d \phi=\frac{1}{2^{2 m}} \sum_{k=0}^{m}\left(\begin{array}{c}
2 m+1 \\
k
\end{array}\right) \frac{\sin \left[(2 m-2 k+1) \frac{\epsilon}{2}\right]}{2 m-2 k+1}
$$

and

$$
\int_{0}^{\frac{\epsilon}{2}} \cos ^{2 m} \phi d \phi=\frac{1}{2^{2 m}}\left(\begin{array}{c}
2 m \\
m
\end{array}\right) \frac{\epsilon}{2}+\frac{1}{2^{2 m-1}} \sum_{k=0}^{m-1}\left(\begin{array}{c}
2 m \\
k
\end{array}\right) \frac{\sin \left[(2 m-2 k) \frac{\epsilon}{2}\right]}{2 m-2 k}
$$

In the sequel, we examine the case $n=2 m+1$. The case $n=2 m$ can be analyzed in the same way so we omit it, for brevity. We see that

$2 \int_{0}^{\frac{\epsilon}{2}} \cos ^{n-1} \phi d \phi=2 \int_{0}^{\frac{\epsilon}{2}} \cos ^{2 m} \phi d \phi=\frac{1}{4^{m}} \frac{(2 m) !}{(m !)^{2}} \epsilon+\frac{1}{4^{m-1}} \sum_{k=0}^{m-1} \frac{(2 m) !}{k !(2 m-k) !} \frac{\sin \left[(2 m-2 k) \frac{\epsilon}{2}\right]}{2 m-2 k}$

We are eventually interested in a small $\frac{\epsilon}{2}$-neighborhood of the equator, with $\epsilon$ chosen to satisfy

$$
|(m-k) \epsilon| \ll 1, \quad \text { as } m \rightarrow \infty
$$

so a Taylor series expansion of the sine function gives, to first order in $(m-k) \epsilon$

$$
2 \int_{0}^{\frac{\epsilon}{2}} \cos ^{2 m} \phi d \phi=\frac{1}{4^{m}} \frac{(2 m) !}{(m !)^{2}} \epsilon+\frac{1}{4^{m-1}} \sum_{k=0}^{m-1} \frac{(2 m) !}{k !(2 m-k) !} \frac{(2 m-2 k) \frac{\epsilon}{2}}{2 m-2 k}
$$

We then use the well-known observation that as $m$ increases $m$ ! increases much faster, so the maximum of $\left(\begin{array}{c}2 m \\ k\end{array}\right)$ is attained when $k=m$, which results in

$$
2 \int_{0}^{\frac{\epsilon}{2}} \cos ^{2 m} \phi d \phi \leq \frac{1}{4^{m}} \frac{(2 m) !}{(m !)^{2}} \epsilon+\frac{2}{4^{m}}(m-1) \frac{(2 m) !}{(m !)^{2}} \epsilon
$$

which eventually gives

$$
2 \int_{0}^{\frac{\epsilon}{2}} \cos ^{2 m} \phi d \phi \leq \frac{2 m-1}{4^{m}} \frac{(2 m) !}{(m !)^{2}} \epsilon
$$


We express the factorial through the gamma function $\Gamma(x)$ as $[12] \Gamma(m+1)=m$ ! and can rewrite (17) as

$$
2 \int_{0}^{\frac{\epsilon}{2}} \cos ^{2 m} \phi d \phi \leq \frac{2 m-1}{4^{m}} \frac{\Gamma(2 m+1)}{[\Gamma(m+1)]^{2}} \epsilon
$$

Using

$$
\Gamma(x+1)=x \Gamma(x), \quad x \in \mathbb{C} \backslash\{-1,-2, \ldots\}
$$

we find

$$
2 \int_{0}^{\frac{\epsilon}{2}} \cos ^{2 m} \phi d \phi \leq \frac{4 m-2}{m 4^{m}} \frac{\Gamma(2 m)}{[\Gamma(m)]^{2}} \epsilon
$$

Substituting $m=(n-1) / 2$, we get

$$
2 \int_{0}^{\frac{\epsilon}{2}} \cos ^{n-1} \phi d \phi \leq \frac{n-2}{(n-1) 2^{n-3}} \epsilon \frac{\Gamma(n-1)}{\left[\Gamma\left(\frac{n-1}{2}\right)\right]^{2}}
$$

It is well-known that

$$
\operatorname{vol}\left(S^{n-1}\right)=\frac{2 \pi^{\frac{n}{2}}}{\Gamma\left(\frac{n}{2}\right)}
$$

which gives

$$
\frac{\operatorname{vol}\left(S^{n-1}\right)}{\operatorname{vol}\left(S^{n}\right)}=\frac{\Gamma\left(\frac{n+1}{2}\right)}{\Gamma\left(\frac{n}{2}\right) \Gamma\left(\frac{1}{2}\right)}
$$

Combining (10) and (23), we find

$$
\frac{\operatorname{vol}\left(U_{\epsilon / 2}\left(S^{n-1}\right)\right)}{\operatorname{vol}\left(S^{n}\right)} \leq \frac{n-2}{(n-1) 2^{n-3}} \epsilon \frac{\Gamma\left(\frac{n+1}{2}\right) \Gamma(n-1)}{\Gamma\left(\frac{1}{2}\right) \Gamma\left(\frac{n}{2}\right)\left[\Gamma\left(\frac{n-1}{2}\right)\right]^{2}}
$$

Using once more (19) and that $\Gamma\left(\frac{1}{2}\right)=\sqrt{\pi},(24)$ reduces to

$$
\frac{\operatorname{vol}\left(U_{\epsilon / 2}\left(S^{n-1}\right)\right)}{\operatorname{vol}\left(S^{n}\right)} \leq \frac{n-2}{2^{n-2}} \frac{\epsilon}{\sqrt{\pi}} \frac{\Gamma(n-1)}{\Gamma\left(\frac{n}{2}\right) \Gamma\left(\frac{n-1}{2}\right)}
$$

Substituting the Legendre duplication formula [12]

$$
\Gamma(2 x)=\frac{2^{2 x-1}}{\sqrt{\pi}} \Gamma(x) \Gamma\left(x+\frac{1}{2}\right)
$$

in $(25)$, we find

$$
\frac{\operatorname{vol}\left(U_{\epsilon / 2}\left(S^{n-1}\right)\right)}{\operatorname{vol}\left(S^{n}\right)} \leq \frac{(n-2) \epsilon}{\pi}
$$

We have already assumed in (14) that, essentially $|n \epsilon| \ll 1$. In this approximation, we find

$$
\lim _{n \rightarrow \infty} \frac{\operatorname{vol}\left(U_{\epsilon / 2}\left(S^{n-1}\right)\right)}{\operatorname{vol}\left(S^{n}\right)}=0
$$


This is a result that someone intuitively expects to hold. The volume of a small tubular neighborhood of the equator is much much smaller than the volume of the whole sphere, even as $n$ increases without an upper bound. Revisiting this derivation we see at least two points which are not quite satisfactory. First, we were "too generous" in our attempts to find an upper bound for $2 \int_{0}^{\frac{\epsilon}{2}} \cos ^{2 m} \phi d \phi$ appearing in (10). We replaced $\sin [(2 m-2 k) \epsilon / 2]$ with its first order Taylor series approximation $(2 m-2 k) \epsilon / 2$ in (15). Second, we uncritically substituted all combinatorial terms $\left(\begin{array}{c}2 m \\ k\end{array}\right)$ with their maximum $\left(\begin{array}{c}2 m \\ m\end{array}\right)$ in (16). The result is a very crude upper bound in (27), which is not sufficiently sensitive to distinguish small deviations from zero of $\frac{\operatorname{vol}\left(U_{\epsilon / 2}\left(S^{n-1}\right)\right)}{\operatorname{vol}\left(S^{n}\right)}$, in case they exist.

\section{Relative VOLUMES OF SPHERES; SECOND ORDER APPROXIMATION}

The state of affairs changes dramatically by sharpening the upper bound of (13). This is done by working at a level of accuracy equivalent to a second order approximation of $\sin (m-k) \epsilon$, which would be naively expected to vanish. The approach of the last few paragraphs, which relies on the explicit calculation of the volumes that we want to compare from the outset, cannot be straightforwardly extended to help us reach our goal. This is due to the fact that there is no obvious better upper bound either for $\sin (m-k) \epsilon$

or for $\left(\begin{array}{c}2 m \\ k\end{array}\right)$ that can sharpen the inequality (16) which we need to use. A second-order approximation in its argument exists only for a cosine, but not for a sine function. The logic that we follow is: we first find a second-order upper bound for the cosine function appearing in (10), and only then we perform the required integrations.

One works as follows [9]. As in (6), let $B(x, \epsilon)=\left\{y \in S^{n}: \rho(y, x)<\epsilon\right\}$. This represents a "cap" of radius $\epsilon$ on $S^{n}$. Instead of the relative volume of the "equatorial ring" $\frac{\operatorname{vol}\left(U_{\epsilon / 2}\left(S^{n-1}\right)\right)}{\operatorname{vol}\left(S^{n}\right)}$ that we considered in $(10)$, let us consider now

$$
\frac{\operatorname{vol}\left(B\left(x, \frac{\pi}{2}+\epsilon\right)\right)}{\operatorname{vol}\left(S^{n}\right)}
$$

This represents the percentage of the total volume that is occupied by spherical "cap" encompassing the equator. We try to find a bound for

$$
\alpha(\epsilon, n)=1-\frac{\operatorname{vol}\left(B\left(x, \frac{\pi}{2}+\epsilon\right)\right)}{\operatorname{vol}\left(S^{n}\right)}
$$

This function expresses the "percentage" of the volume of $S^{n}$ which is left out after subtracting the volume of the spherical "cap" defined above from $S^{n}$. According to the 
general volume formulae $(7),(8),(22), \quad \alpha(\epsilon, n)$ can be expressed as

$$
\alpha(\epsilon, n)=\frac{\int_{\frac{\pi}{2}+\epsilon}^{\frac{\pi}{2}} \sin ^{n} \theta d \theta}{\int_{0}^{\pi} \sin ^{n} \theta d \theta}
$$

Substituting $\phi=\theta-\frac{\pi}{2}$, as in (9) and setting $I_{n}=\int_{0}^{\pi} \cos ^{n} \phi d \phi$, (31) can be rewritten as

$$
\alpha(\epsilon, n)=\frac{\int_{\epsilon}^{\frac{\pi}{2}} \sin ^{n} \phi d \phi}{\int_{0}^{\pi} \sin ^{n} \phi d \phi}
$$

Changing the variable of integration to $w=\phi \sqrt{n}$, we get

$$
\alpha(\epsilon, n)=\frac{1}{2 I_{n} \sqrt{n}} \int_{\epsilon \sqrt{n}}^{\frac{\pi}{2} \sqrt{n}} \cos ^{n}\left(\frac{w}{\sqrt{n}}\right) d w
$$

Comparing the Taylor series expansions of $\cos w$ and of $\exp \left(-w^{2} / 2\right)$ in $\left[0, \frac{\pi}{2}\right]$, we see that $\cos w \leq \exp \left(-w^{2} / 2\right)$, so (33) gives

$$
\alpha(\epsilon, n) \leq \frac{1}{2 I_{n} \sqrt{n}} \int_{0}^{\left(\frac{\pi}{2}-\epsilon\right) \sqrt{n}} e^{-\frac{w^{2}}{2}} d w
$$

By setting $t=w+\epsilon \sqrt{n}$ we find that

$$
\alpha(\epsilon, n) \leq \frac{1}{2 I_{n} \sqrt{n}} \int_{\epsilon \sqrt{n}}^{\frac{\pi}{2} \sqrt{n}} e^{-\frac{(t+\epsilon \sqrt{n})^{2}}{2}} d t
$$

which implies

$$
\alpha(\epsilon, n) \leq \frac{e^{-\frac{\epsilon^{2} n}{2}}}{2 I_{n} \sqrt{n}} \int_{0}^{\infty} e^{-\frac{t^{2}}{2}} d t
$$

which using the fact that $\int_{0}^{\infty} \exp \left(-\frac{t^{2}}{2}\right) d t=\sqrt{\frac{\pi}{2}}$ gives

$$
\alpha(\epsilon, n) \leq \frac{1}{I_{n} \sqrt{n}} \sqrt{\frac{\pi}{8}} \exp \left(-\frac{\epsilon^{2} n}{2}\right)
$$

The straightforwardly derived recursion relation $(n+2) I_{n+2}=(n+1) I_{n}$ implies that

$$
\sqrt{n+2} I_{n+2}=\frac{n+1}{\sqrt{n+2}} I_{n} \geq \sqrt{n} I_{n}
$$

Obviously $I_{1} \geq 1$ and $\sqrt{2} I_{2} \geq 1$, therefore $\sqrt{n} I_{n} \geq 1, \forall n \in \mathbb{N}$, which gives

$$
\frac{\operatorname{vol}\left(B\left(x, \frac{\pi}{2}+\epsilon\right)\right)}{\operatorname{vol}\left(S^{n}\right)} \geq 1-\sqrt{\frac{\pi}{8}} \exp \left(-\frac{\epsilon^{2} n}{2}\right)
$$


Let $U \subset S^{n}$ with $\operatorname{vol} U / \operatorname{vol}\left(S^{n}\right) \geq 1 / 2$ and $\epsilon>0$. For any $\epsilon$-neighborhood $U_{\epsilon}$ of $U$, the classical Lévy-Gromov isoperimetric inequality for $S^{n}[3]-[7]$ states that

$$
\frac{\operatorname{vol}\left(U_{\epsilon}\right)}{\operatorname{vol}\left(S^{n}\right)} \geq \frac{\operatorname{vol}\left(B\left(x, \frac{\pi}{2}+\epsilon\right)\right)}{\operatorname{vol}\left(S^{n}\right)}
$$

Combining the above statements we arrive at the concentration inequality

$$
\frac{\operatorname{vol}\left(U_{\epsilon}\left(S^{n-1}\right)\right)}{\operatorname{vol}\left(S^{n}\right)} \geq 1-\sqrt{\frac{\pi}{8}} \exp \left(-\frac{\epsilon^{2} n}{2}\right)
$$

Its implication is immediate, although counter-intuitive: as the dimension $n$ of the unit sphere $S^{n}$ becomes very large, all its volume is concentrating in a thin ring of width $\epsilon \sim \frac{1}{\sqrt{n}}$ around its equator. An intuitive objection to this conclusion is the following; $S^{n}$ has an infinite number of great circles $S^{n-1}$ at the $\epsilon$-neighborhood in each of which the volume is concentrated, according to (41). Let's pick one such great circle with its corresponding $\epsilon$-neighborhood. Each $\epsilon$-neighborhood of every other great circle should have volume zero, a fact that appears to contradict (41) which does not discriminate in favor of one among the great circles and their $\epsilon$-neighborhoods. This inequality also seems to violate the sphere being a symmetric space, consequently its points being connected through the transitive action of its isometry group. Since the volume is a Hausdorff measure directly derived from the round metric $\rho$ of $S^{n}$, it appears that is should also possess the symmetries of $\rho$.

This apparent contradiction can be partly resolved, if we consider what happens to the volume of $S^{n}$ for large $n$. Substituting the Stirling approximation

$$
\Gamma(x+1) \sim \sqrt{2 \pi x}\left(\frac{x}{e}\right)^{x}, \text { for } x \rightarrow \infty
$$

in (22) and using that

$$
\lim _{x \rightarrow \infty}\left(1+\frac{k}{x}\right)^{x}=e^{k}, \quad \forall k \in \mathbb{R}
$$

we find

$$
\operatorname{vol}\left(S^{n-1}\right) \leq \sqrt{2} \pi\left(\frac{2 e \pi}{n^{1-\frac{1}{n}}}\right)^{\frac{n}{2}}
$$

which implies that

$$
\lim _{n \rightarrow \infty} \operatorname{vol} S^{n-1}=0
$$

as fast as $n^{-\frac{n}{2}}$. A volume approaching zero means that the $n$-dimensional manifold in question, $S^{n}$ in our case, "collapses" to one of lower dimension, from a measuretheoretical viewpoint. This is odd, considering that the metric properties of $S^{n}$ remain 
those of an $n$-dimensional space. By comparing (41) and (45), we conclude that the vol-

ume of $S^{n}$ decreases much faster toward zero than the relative volume $\frac{\operatorname{vol}\left(U_{\epsilon}\left(S^{n-1}\right)\right)}{\operatorname{vol}\left(S^{n}\right)}$ so, measure-theoretically, $S^{n}$ is effectively reduced to $S^{n-1}$. Finally, it is probably worth mentioning, for completeness, that this volume decline of $S^{n}$ toward zero as a function of $n$ does not contradict the topology of $S^{n}$ : indeed, one can argue [13] that the infinite sphere $S^{\infty}$ is contractible, hence topologically trivial. The discrepancy between our intuition and the results of this section should make us be very careful when trying to extend already understood concepts of a fixed dimension, to the case of such a dimension approaching infinity. Indeed, as we just saw, in the latter case, which is a particular example in the asymptotic theory of normed spaces, our low-dimensional geometric intuition may misguide us if we try to make predictions or support arguments by relying on it too much. This is not totally surprising as geometry, proper, actually refers to spaces of fixed (Hausdorff) dimension. By contrast, we are dealing with calculations of non-trivial limits of sequences of inequalities in spaces where the dimension is a free parameter, and not totally surprisingly new phenomena occur in such limits.

It may also be worth noticing that in (41) the relative volume of the tubular neighborhood of the equator declines in a Gaussian way as a function of $\epsilon$. Because of this, (41) can be seen as providing a geometric model for the law of large numbers [4].

\section{Applichtion to an ideal gas}

Consider a gas, in one spatial dimension for simplicity, of $n-1$ identical particles of unit mass, with momenta $p_{i}, i=1, \ldots, n-1$ and interacting with each other through the potential $\Phi\left(x_{1}, \ldots, x_{n-1}\right)$. The Hamiltonian of such a system is given by

$$
\mathcal{H}=\sum_{i=1}^{n-1} \frac{p_{i}^{2}}{2}+\Phi\left(x_{1}, \ldots, x_{n-1}\right)
$$

We put this gas inside a thermally isolated one-dimensional box (a line segment) of volume (length) $V$, whose walls (endpoints) are perfectly reflecting. The equilibrium statistical behavior of such a system is determined by calculating the micro-canonical distribution

$$
\tau\left(p_{i}, x_{j}\right): \text { const, } \quad 0 \leq i, j \leq n-1
$$

on the constant energy $E$ hyper-surface of phase space with the normalization

$$
\int_{\mathcal{H}=E} \tau\left(p_{i}, x_{j}\right)=1
$$


For an ideal gas

$$
\Phi\left(x_{1}, \ldots, x_{n-1}\right)=0
$$

Then, the phase space of the system is $V^{n-1} \times \mathbb{R}^{n-1}$. We have used the fact that the total energy $E$ of the system is conserved and is given by

$$
\mathcal{H}=\sum_{i=1}^{n-1} \frac{p_{i}^{2}}{2}=E
$$

and assuming, for simplicity, that $E=1 / 2$ energy unit, the phase space of the system is reduced to $V^{n-1} \times S^{n-2}$. We have tacitly assumed that there are no other integrals of motion except the total energy $E$. As a result, the phase space of the system becomes $V^{n-1} \times S^{n-2}$ but cannot be further reduced due to the lack of such another conserved quantity. The part of phase space giving a non-trivial contribution to the physical behavior of the system is $S^{n-2}$. This is equivalent to reducing the micro-canonical distribution dependence on the momenta only

$$
\tau\left(p_{i}, x_{j}\right)=\tau\left(p_{i}\right)
$$

Indeed, the factors due to $V^{n-1}$ are integration constants, which can be safely omitted in the subsequent arguments.

We now add one more particle to the gas of the $n-1$ particles. We assume that this particle which we add is stationary in the frame or reference of the line segment. The total energy of the system will remain $E$ and its phase space will now become $V^{n} \times S^{n-1}$. Due to collisions with the other particles, the one that we added will eventually acquire the same average kinetic energy $\bar{E}=\frac{E}{n}$ as the other particles (equipartition). We consider the non-trivial part of the micro-canonical expectation value

$$
\left\langle p_{i}^{2}\right\rangle=\int_{S^{n-1}} p_{i}^{2} \tau\left(p_{j}\right) d \omega
$$

where $d \omega$ is the (normalized) Lebesgue measure on $S^{n-1}$ associated to the round metric. Since $S^{n-1}$ is an isotropic space, we have

$$
\left\langle p_{i}^{2}\right\rangle=\int_{S^{n-1}} \frac{1}{n} \sum_{i=1}^{n} p_{i}^{2} \tau\left(p_{j}\right) d \omega
$$

which, upon integration and after using (47) and (48), gives

$$
\left\langle p_{i}^{2}\right\rangle=\frac{1}{n}
$$


The isotropy of $S^{n-1}$ also implies that $\left\langle p_{i}\right\rangle=0$, which gives for the standard deviation $\sigma_{n}$ of the momentum of each particle of the $n$-particle gas

$$
\sigma_{n}=\frac{1}{\sqrt{n}}
$$

This result can be interpreted as follows: as the number of particles $n$ of the gas increases, there is an uncertainty $\sigma_{n}=n^{-\frac{1}{2}}$ in the value of the momentum of each particle. If we add a stationary particle, the $n$-particle gas will be indistinguishable from the $(n+1)$-particle gas within an accuracy which is determined by $\sigma_{n}$. The perturbation that the system will undergo by the introduction of such a stationary particle will not be detectable within the given level of accuracy. This is despite the fact that the phase spaces $S^{n-1}$ and $S^{n}$ are not even homeomorphic, let alone diffeomorphic. We can easily check that $S^{n}$ and $S^{n-1}$ are topologically, hence differentiably, distinct by comparing their corresponding cohomology groups [10], for instance. The relation of the metric properties of $S^{n}$ and $S^{n-1}$ can be found as follows: Let $d s_{n-1}, d s_{n}$ denote the Riemannian round metrics of $S^{n-1}, S^{n}$ respectively. Then topologically $S^{n}=\Sigma\left(S^{n-1}\right)$ where $\Sigma$ in this relation indicates the spherical suspension [13]. Because of this suspension, we come to suspect that $d s_{n}$ and $d s_{n-1}$ are related by

$$
d s_{n}=[0, \pi] \times_{\sin \theta} d s_{n-1}
$$

In (56), $\times_{\sin \theta}$ indicates a warped product with function $\sin \theta$. We can rewrite (56), in a slightly more familiar form as

$$
d s_{n}^{2}=(d \theta)^{2}+\sin ^{2} \theta d s_{n-1}^{2}
$$

which can be straightforwardly seen to be correct. The case of a particle added to the system is expressed by a slowly varying value of $\theta$ around $\theta=\frac{\pi}{2}$. Such an approximation gives $d \theta=0$ and up to first order in $\theta$ (which is proportional to $\sigma_{n}$ ) we find

$$
d s_{n}=d s_{n-1}
$$

which, in turn, implies that the immersion $i: S^{n-1} \hookrightarrow S^{n}$ is locally distance-preserving. Therefore, within a momentum uncertainty $\sigma_{n}$, it is impossible to find locally any difference between the metric spaces $S^{n}$ and $S^{n-1}$. Roughly speaking, this amounts to stating that if someone looks at $S^{n}, S^{n-1}$ with eyeglasses whose resolution is larger than $\sigma_{n}=n^{-\frac{1}{2}}$, then they appear to be identical, as metric spaces, in a tubular neighborhood 
of $S^{n-1}$.

It is probably worth mentioning at this point that similar arguments can be presented [9] for systems whose configuration space $\mathcal{M}_{l}$ is $l \in \mathbb{N}$ copies of $S^{n}$, namely

$$
\mathcal{M}_{l}=\underbrace{S^{n} \times S^{n} \times \ldots \times S^{n}}_{l \text { times }}
$$

A physical example can be provided by $l$ different ideal gases, each one containing $n$-particles which coexist in volume $V$. In such a case the phase space volume is the product of the Riemannian volumes on each copy of $S^{n}$. Let $x=\left(x_{1}, x_{2}, \ldots, x_{l}\right)$ and $y=\left(y_{1}, y_{2}, \ldots, y_{l}\right)$ be two elements of $\mathcal{M}_{l}$. The distance $\rho_{\mathcal{M}_{l}}(x, y)$ between $x, y \in \mathcal{M}_{l}$ is chosen to be

$$
\rho_{\mathcal{M}_{l}}(x, y)=\left(\sum_{i=1}^{l} \rho\left(x_{i}, y_{i}\right)^{2}\right)^{\frac{1}{2}}
$$

as one would normally expect for the Cartesian product of metric spaces. It turns out, not too surprisingly, that the constants in the Lévy-Gromov inequality are the same as the ones entering (41) and the interpretation of momentum localization is analogous to that case.

\section{Discussion AND CONCLUSIONS}

By using analytic and geometric arguments, we have described the behavior of a special case of an ideal gas, and determined the metric and measure-theoretical behavior of its phase space in the thermodynamic limit. As a by-product of this approach, we obtained an upper bound for the rate of approach of this system to equilibrium. Although these analytic results have been known for a while [3], we have not been able to find a reference that makes explicit the approach, connections and statistical implications that we make in the present letter.

The measure concentration that we observe in the case at hand constitutes, in a way, a form of symmetry breaking. Indeed, we start with $S^{n}$ (vacuum of the "unbroken phase") equipped with the round metric whose isometry group is $O(n)$. As $n$ increases, the Haar measure of $S^{n}$ localizes in a tubular neighborhood of $S^{n-1}$ (vacuum of the "broken phase"), which has the smaller isometry group $O(n-1)$. In our case, the parameter describing the concentration of measure, which plays the role that the order parameter plays 
in the usual symmetry breaking, is the dimension of the phase space $n$ of the system. From the viewpoint of measure theory, a higher dimension, which physically describes a larger number of degrees of freedom, does not lead to a more complicated behavior, a fact which is well-known and extensively employed when constructing the thermodynamic limit of a statistical system. This behavior is also evident in the simplifications occurring during the $1 / N$ expansion of the $O(N)$ models [14] and the associated gauge theories with a large number $N_{c}$ of colors [15], [16]. Needless to say, the thermodynamic limit of a statistical system also leads to such simplifications. Actually, part of the goal of this paper was the description, in a more geometric language, of the way some systems, like our specifically chosen example of an ideal gas, behave when considering their thermodynamic limit.

For the future, we would like to know whether the present analysis can be extended to cover interacting statistical systems. This would certainly be of much more interest than the example we have presented in this paper. The difficulty with interacting systems is threefold: First, they have phase spaces that are considerably more complicated than those of free systems. Second, checking the ergodicity of the Hamiltonian flow on such phase spaces, which provides the dynamical foundation of statistical mechanics and its mixing properties which determine the rate of approach of the system toward equilibrium is practically impossible for all but the simplest cases. Third, explicit calculation of the canonical partition function of most interacting systems is practically intractable, generically, except through perturbation theory or lattice approximations. Even if such calculations were possible however, it is unlikely that the final result could be expressed in terms of purely, or easily identifiable, geometric quantities as in the example we have analyzed. Then, instead of the relatively straightforward analytic and geometric arguments presented in this paper, the full machinery of measure theory would have to be used, a fact that would enormously complicate the analysis and detract from the underlying geometric structures. In the spirit of the present paper, considerable progress in generalizing the arguments and applying them to the case of spin glasses was made in [17].

ACKNOWLEDGEMENT: We are grateful to the referee whose criticisms helped correct some mistakes of an earlier version of the manuscript and whose comments helped considerably elucidate several points of the exposition. 


\section{REFERENCES}

[1] M. Ledoux, The Concentration of Measure Phenomenon AMS, Providence (2001)

[2] V.D. Milman, Funct. Anal. Appl. 5, 28 (1971)

[3] P. Lévy, Problemes Concretes d'Analyse Fonctionelle, Gauthier-Villars, Paris (1951)

[4] M. Gromov, Metric Structures for Riemannian and Non-Riemannian Spaces, Birkhäuser, Boston (1999)

[5] Yu.D. Burago, V.A. Zalgaller, Geometric Inequalities, Springer, Berlin (1988)

[6] I. Chavel, Isoperimetric Inequalities, Camb. Univ. Press, Cambridge (2001)

[7] M. Gromov, Isoperimetric Inequalities in Riemannian Manifolds, Appendix I of Ref.[9]

[8] T. Figiel, J. Lindenstrauss, V. Milman, Acta Math. 139, 53 (1977)

[9] V.D. Milman, G. Schechtman, Asymptotic Theory of Finite Dimensional Normed Spaces, Lecture Notes in Mathematics 1200, Springer, Berlin (1986)

[10] V.D. Milman, Astérisque, 157-8, 273 (1988)

[11] N. Kalogeropoulos, Int. J. Mod. Phys. A 23, 509 (2008)

[12] I.S. Gradshteyn, I.M. Ryzhik, Table of Integrals, Series and Products, 5th Edition, Academic Press, London (1984)

[13] E. H. Spanier, Algebraic Topology, McGraw Hill, New York (1966)

[14] G. 't Hooft, Large N, arXiv:hep-th/0204069

[15] G. 't Hooft, Confinement at Large $N_{c}$, Presented at "Large N QCD", Trento, Italy, 5-9 July 2004, arXiv:hep-th/0408183

[16] M. Moshe, J. Zinn-Justin, Phys. Rep. 385, 69 (2003)

[17] M. Talagrand, IHES Publications, 81, 73 (1995) 